\title{
Research on the Development of Pattern Recognition and Applications on Digital Music Recognition and Creation
}

\author{
Sui Wu \\ Hunan City University, \\ Yiyang,Hunan,413000 China
}

\begin{abstract}
In this paper, we conduct general research on the development of pattern recognition and applications on the digital music recognition and creation. Voice is the most convenient tool in human communication and exchange of basic information and the most important media, therefore, speech recognition has a very important position in multimedia technology. Speech recognition technology is a collection of acoustics, phonetics, such as the information processing and artificial intelligence, a comprehensive technology in the field of application is very wide, has been the hot topics in the study of people. The research result has been widely used in every field of human society, and the results of this study would provide great help for the audio information retrieval. Under this background, we propose the novel methods for digital music that is meaningful.
\end{abstract}

Keywords- Pattern Recognition, Digital Music, Recognition and Creation, Applications, Review.

\section{Introduction}

Sounds very abstruse, music aesthetics is also very professional, closer to the abstract theories level, some distance from the music appreciation and music teaching activities. Music aesthetics is actually in the side, namely the musicians and music appreciation, is as long as there are correlation and music activities there is bound to be and music aesthetics. Especially for musicians, including the creation teaching music, music performance, music, but also a are indispensable from theory to practice theory armed and practice activities. Willing to aesthetic, as depth of original aesthetic correctly, musicians and music for each film appreciation is motivated the source of the second creation practice. Specific to music the music creator and carrier groups speaking, for the music creation to his music aesthetics based on music aesthetic theory, in accord with the basic theory of law to pursue its own innovation, on the basis of the formation of personal style, more influential works as the music performers in the music performance in the unity of the need to follow the faithful to the original work and performance to create, to grasp the historical music style and the spirit of the age the same, pay attention to the unity of performance skills and artistic aesthetic principles to exercise performance [1-3].

Computer music production technology and modern multimedia system is the crystallization of the development of science and technology, also an important part of the environment of digital music technology and hardware support. Computer music production technology refers to the use of related hardware and software for editing and finishing technology of music information. Computer music production technology in China has for the majority of music workers and loved by the music lovers. Both music film and television stage, advertising, music, computer music in fact has a major position. Its advantage is: easy to modify, with traditional instruments and the band cannot reach the sound, with excellent quality and general wide dynamic characteristics. Digital music and computer music environment with the development of the computer and the Internet, we realize that they can also be applied in the teaching of the music studied composition with related equipment harmony, practice ear and production of printed music and all aspects of related disciplines.

In order to be able to better music retrieval, the early stage of the work is to classify a large 
number of music files. If the music classification is a good comment, so customers can easily find them very much interested in music, and the different properties between different types of music, music so will classify annotation is also in order to be able to manage them more effectively. Because the music classification annotation is seriously influenced by the subjective factors, so before this work finished basic is artificial. But faced with huge amounts of music files, artificial classification of annotation ability is limited, and this kind of practice violates the efficient retrieval and management of music files, how to automatically and accurately classify music that has become the main way to solve these problems. Experts and scholars in the field of the music information retrieval will be more and more attention on how to better music classification, many new techniques are used to finish the task, make the music classification gained unprecedented development [4-5].

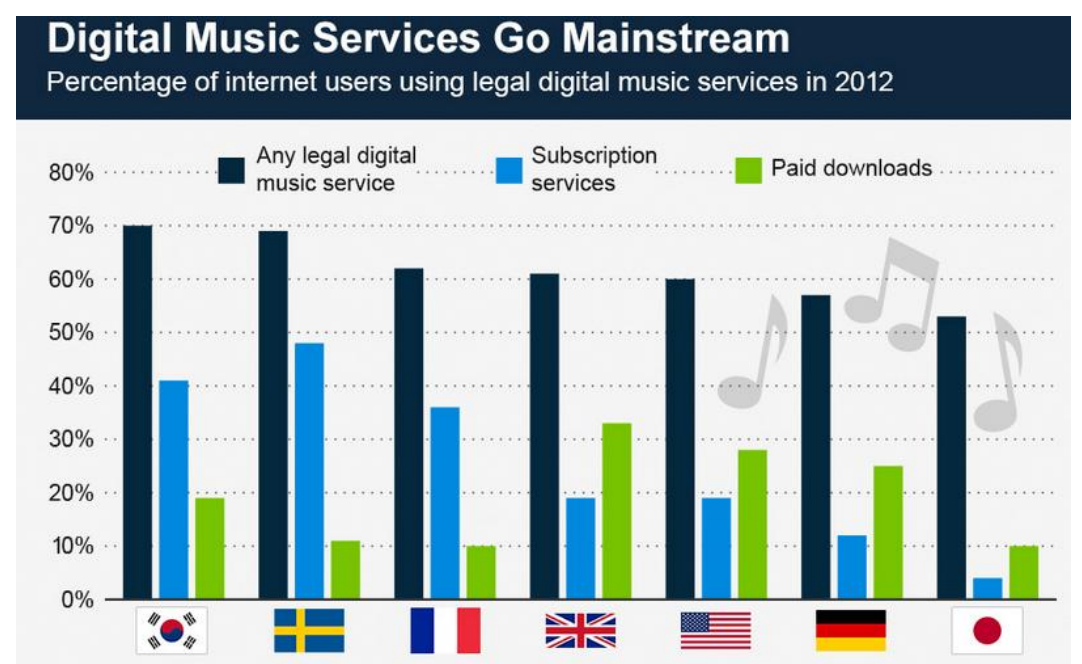

Figure 1. The Statistical Data of the Digital Music Marcket

In this paper, we conduct research on the development of pattern recognition and applications on digital music recognition and creation. Digital music teaching methods including multimedia music education teaching means, digital music production means and network music education teaching method and so on three areas. With the high-speed development of electronic science and technology and the education level unceasing enhancement, the digital teaching method as an auxiliary teaching method is widely used in vocal music teaching. In the later sections, we will discuss in detail.

\section{The Proposed Methodology}

The Characteristics of the Digital Music. With the progress of science and technology and social development, especially the rise of modern communication technology and application of linguistic study has been involved in many fields of natural science, such as the physical and acoustic phonetic research, digital transmission of sound language culture research, voice information evolutionary computation of the study of the language cognition and coding, language pronunciation physiological research evolution of human language, language physical and emotional songs, drama and original ecological vocal music study, etc. Obviously, the study of language and the oral culture has gradually been more scientific. Listening training how to adapt to the development of digital music environment could be generally summarized as the follows. (1) Indispensable to teaching in the environment of the digital technology and hardware support for establishment of the auditory training classroom. Digital music 
environment essential technology and hardware support. The use of modern computer music production technology and only under the support of related hardware and software, to make music, sound information for editing and finishing so the premise of the digital multimedia teaching, must establish the digital multimedia system is listening training classroom. (2) Core recording auditory training in the classroom, not only can aims thousands of different hearing the music, and you can also do compose sing, solfeggio, improve cement listening training as this form of used to overcome the blindness of students before training and boring, and basic rich content of the training and scientific detection methods to enhance the training of interest [6-7].

To explore the value of music under the digital media refactoring inevitably involves how to treat traditional music and modern music the two problems. For the digital music, we inevitably to achieve supreme purport and state of art and yearning, based on the standard, think of it this way: true than false more valuable, more valuable than mediocre. Only in this way, the future of digital music art and the relationship of science and technology will show the technology of art and art technology.

Look from the evolution law of music history, digital music should have broad prospect, especially with the development of digital audio and the video and other multimedia, digital music will be from multivariate to more diverse. In addition, from a technical level, the ultimate goal of development of digital technology is the "simulation", also reflect simulation model of traditional music to maximize positive demand, reflect the creation value maximization, embodies the "real" in the "virtual", make the human to realize the most convenient process of music creation and appreciation of music creation space, tone change, harmony experience, deductive method, mode of transmission to unprecedented height and width, let music art and the perfect unity of science and technology become a reality.

The humanistic spirit and value of digital media background music refactoring, should not take it as the music of dissent. Like impressionist had just appeared, it is not a disaster, and can think it is a kind of music genre and style, the value of music works reflect more is determined by the cultural, the social and psychological, music works from creation to spread is more depends on the intrinsic value of the music and is not the technology itself [8].

The Pattern Recognition Concepts. From the narrow sense, the so-called mode is mainly refers to describe the structure and the object of interest is quantitative, the combination of the model class is common for most. Pattern recognition is the study of some automatic technology, make the computer on the basis of these technologies and less human intervention for general model assigned to the respective belong in the class can be seen from the description of the concept, the model method of describing the structural description and quantitative description, and the matching method of pattern recognition, including two types of structural pattern recognition and statistical pattern recognition.

In the process need to use some samples for the determination of classification discriminant rule to ensure it is concluded that the classification of the discriminant rules can reduce may occur in the process of recognition error rate as classifier is classified according to conclude discriminant rule to treat general patterns for classification and discrimination and the classification results are output.

After years of research and development, pattern recognition technology has been widely used in artificial intelligence, computer engineering, machine learning, neural biology, medicine, detective and high energy physics, archaeology, geological exploration, weapons of aerospace science and the technology and many 
other important areas that could be summarized as the follows.

- The application of pattern recognition in medicine. In medical field, the pattern recognition not only has a wide range of application, at the same time has a good application prospect, and with the development of health service in our country, many of the need to rely on artificial to complete the work more and more, and it also decides the future development in the medical field, the application of pattern recognition technology will be more deeply and widely.

- The application of pattern recognition in speech recognition. Relative to character recognition based on pattern recognition, based on pattern recognition of speech recognition has greater complexity and difficulty, this is mainly because of basic pattern recognition based on speech recognition is not only to the phonetic features extraction, such as the voice, such as physical process, the structure of general voice at the same time and the physical process of hearing has strongly associated with physical processes.

- Pattern recognition application in modern character recognition. Although Chinese character recognition based on pattern recognition based on with great difficulty, but for that need a lot of language school work, character recognition based on the pattern recognition technology improvement and development will inevitably increase the scope of these departments using pattern recognition and to promote efficiency of department play an important positive role.

The Digital Music Recognition. For music retrieval is based on the content of the music retrieval, through hum a tune melody fragment, extracted from music melody and rhythm feature information such as search, there are very few songs to make use of audio retrieval technique similarity retrieval. Using the relevant technology to achieve similar music retrieval, singers can according to your own voice and style, the use of retrieval system and similar to their own songs, at the same time, the user can automatically from song select their favorite songs, satisfy personalized music recommendation and service. In the following figure, we show the mentioned scenario.

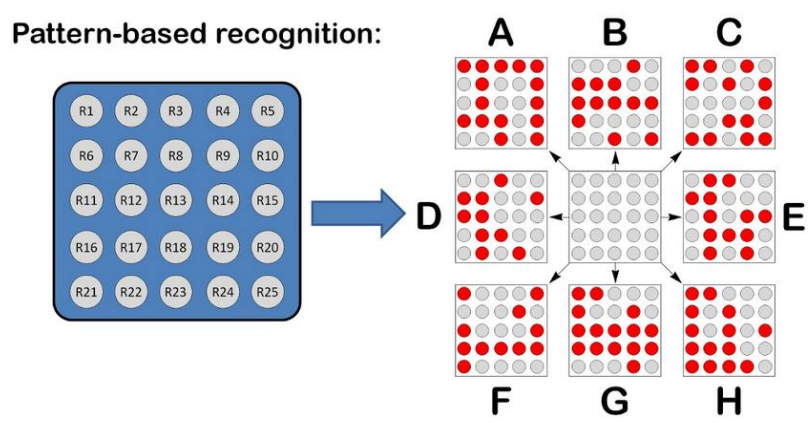

Figure 2. The Demonstration of the Digital Music Recognition Framework

This paper uses integrated former sequence selection as circulation under the framework of static selection strategy. Entered before the idea is based on the order, order before entered is the optimal precision as the criteria for the bottom-up search method, and is applied in many aspects, such as feature selection. Integrated sequence selection method is according to the base classifier before the precision of the size of the difference of sequence comparison before and after the combination if difference increased. The corresponding judgement paradigm could be listed as the follows.

$$
L(\lambda \mid X)=P(X \mid \lambda)=\prod P\left(x_{t} \mid \lambda\right)
$$

The base classifier selection of clustering has to the cycle framework provides a good integration environment, reduce number of candidate base classifier, allowed circulation under the framework of selective strategy to less base classifier for integration, integration achieved good results. Relatively, the target of 
the mentioned issues could be summarized as the following procedure.

\section{(2)}

$$
\arg \max \sum \sum w_{i k} p_{i k}
$$

\section{The Future of the Musical Creation. Music} and painting in theory seems to be two different is not related to art, the former through voice appeals to people's hearing organ, while the latter through the line and color to people's visual organ. But in the real art activities, the so-called painting sense in music or music in painting is often used for the discussion. Like a painting of the word "color" is often appear as music language in the music theory article; Music in the word "rhythm" is often used as painting language. From the perspective of physics, color in the sound of music and painting are a kind of fluctuation as is the sound waves and light waves although both are volatile, but they are very different in nature and the frequency range between [9].

Music creation cannot be separated from culture we live in a society full of culture all are restricted by culture. Musicians can social culture positive reaction. Is one of the main conditions of the music society function, musicians as an individual life in the big family of society, in social family, through a variety of social change, music show his household music means the feeling of this kind of change is an important content of music creation. Music culture has close relation, all music practice activity, is under the background of culture during these activities cannot leave the music culture the relationship between fish and water, so to speak. Music is an important part of culture, the creation of the musician was the reaction of his inner thoughts, this reaction and his era culture background, and the author of a work of literature to understand with a deep culture has a close connection. These relationships of his music creation played a good role, constitutes the main content of the music practice. Therefore, to explore the music practice the relationship between music and culture. For us to a new understanding from the point of view of music is very necessary.

\section{Conclusion}

In this paper, we conduct research on the development of pattern recognition and applications on the digital music recognition and creation. Through the above analysis of the connotation of the music style and discussed, and the trajectory from the history of music anthropology see music style changes on the content, you can see the music style as the time, space, each level factors such as geographical environment, define the concept of constantly enrich and expand its meaning is in constant changing, it along with the development of human knowledge and developing and updating. "Music style" in the anthropology of the music horizon is a continuous development of changing concept and it has the characteristics of open as music in the style of study that is of great value in the music anthropology research basic value and significance. In the recent future, we will verify more related algorithms for music recognition to achieve for better performance.

\section{References}

[1] Aguiar, Luis, and Bertin Martens. "Digital music consumption on the internet: evidence from clickstream data." Information Economics and Policy (2016).

[2] Castro Soeiro, Filipe, Mariana Santos, and José Alves. "Network-based Innovation: The case for Mobile Gaming and Digital Music." European Business Review 28.2 (2016).

[3] Morris, Jeremy W. "Making music behave: Metadata and the digital music commodity." New Media \& Society 14.5 (2012): 850-866.

[4] Sim, Jia-Jia, et al. "Understanding and predicting the motivators of mobile music acceptance-a multi-stage MRA-artificial 
neural network approach." Telematics and informatics 31.4 (2014): 569-584.

[5] Magee, Wendy L. "Using electronic and digital technologies in music therapy: the implications of gender and age for therapists and the people with whom they work." (2014).

[6] Wang, Kai, and Shin-Ting Huang. "How flow experience affects intention to use music streaming service: Model development." Proceedings of the 12th International Conference on Advances in Mobile Computing and Multimedia. ACM, 2014.
[7] Hracs, Brian J. "Cultural intermediaries in the digital age: the case of independent musicians and managers in Toronto." Regional Studies 49.3 (2015): 461-475.

[8] Pearce, Nick, et al. "Digital scholarship considered: How new technologies could transform academic work." in education 16.1 (2012).

[9] Stowell, Dan, and Alex McLean. "Live music-making: A rich open task requires a rich open interface." Music and human-computer interaction. Springer London, 2013. 139-152. 